
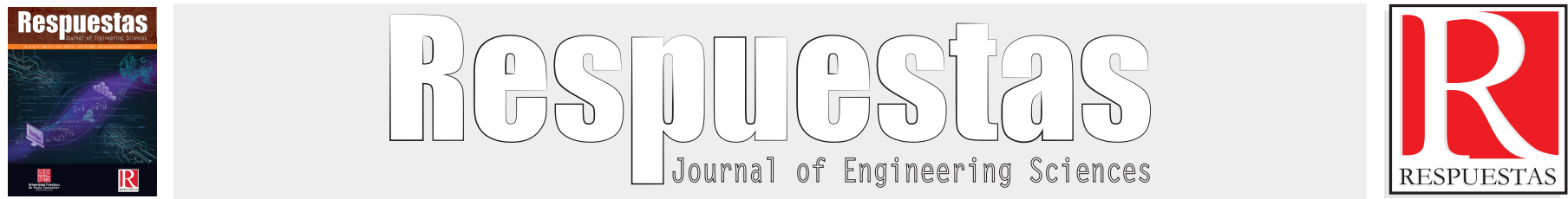

Artículo Original

https://doi.org/10.22463/0122820X.1500

\title{
Control estadístico de procesos en organizaciones del sector servicios
}

\author{
Statistical Process Control in Organizations Services Sector \\ Yenith Cristina Ortiz-González, Ivan Mauricio González-Gaitán. \\ "Magister en calidad y gestión integral, induscris@yahoo.es, orcid.org/0000-0003-3601-6517, Fundación Universidad de América, Bogotá, Colombia. \\ ${ }^{b}$ Especialista en Gerencia de la Calidad, maurogonga@gmail.com, orcid.org/0000-0002-0094-7515, Fundación Universidad de América, Bogotá, Colombia. \\ Cómo citar: Y.C. Ortiz-González y I.M. González-Gaitán, "Control estadístico de procesos en organizaciones del sector servicios", Respuestas, vol. 23, no. S1, \\ pp. 42 - 49, 2018. https://doi.org/10.22463/0122820X.1500
}

Recibido: Febrero 01, 2018; Aprobado: Julio 18, 2018

\begin{tabular}{|c|c|}
\hline & RESUMEN \\
\hline $\begin{array}{l}\text { Calidad } \\
\text { Control estadístico de procesos } \\
\text { Empresas de servicios } \\
\text { Gráficos de control }\end{array}$ & $\begin{array}{l}\text { Antecedentes: Existen casos de estudio individuales que mencionan el uso del control estadístico de procesos, sin embargo no } \\
\text { se ha evidenciado un documento que compile conceptos y aplicación de esta herramienta en empresas de servicios. Como lo } \\
\text { mencionó Zeithalm [1], en su investigación sobre la calidad en los servicios, la información que se encuentra está dedicada, casi } \\
\text { exclusivamente, en la aplicación de productos tangibles. Objetivo: Identificar la aplicación del control estadístico de procesos } \\
\text { en empresas de servicios. Método: Se realizó la búsqueda documental, previa definición del periodo histórico para la correspon- } \\
\text { diente búsqueda, en diferentes bases de datos: Redalyc, Scielo, Ebsco, Ebrary, Proquest, y Virtualpro. Con esta información se } \\
\text { clasificaron los documentos que aplican al tema tratado. Resultados: Se identifican los conceptos relacionados con el control } \\
\text { estadístico de procesos, y su aplicación en empresas de diferentes sectores de servicios (educación, financiero, entre otros). } \\
\text { Conclusión: El control estadístico de procesos es una herramienta aplicable en cualquier tipo de sector económico, incluyendo } \\
\text { empresas de servicios, permitiendo mejorar el desempeño de los procesos que la conforman y, seguramente, aumentar la } \\
\text { satisfacción del cliente. }\end{array}$ \\
\hline
\end{tabular}

\section{ABSTRACT}

Keywords:

Background: There are individual case studies that mention the use of statistical process control, however has not been shown a

Quality

Statistical process control document that compile concepts and application of this tool in business services. As mentioned Zeithalm [1], in his research on the quality of services, the information is is dedicated almost exclusively in implementing tangible products. Objective: Identify the application of statistical process control in service companies. Methods: documentary search was conducted, prior definition of the historical period for the corresponding search in different databases: Redalyc, Scielo, Ebsco, Ebrary, Proquest, and Virtualpro. With this information the documents were classified apply to the subject treated. Results: The concepts related to statistical process control are identified, and their application in companies of different sectors (education, financial, etc.). Conclusions: Statistical process control is a tool applicable in any economic sector, including service companies, allowing improve the performance of the processes that shape it and certainly increase customer satisfaction.

\section{Introducción}

Las empresas, tanto de servicios como de manufactura, tienen un aspecto clave en común: los clientes y su nivel de satisfacción con respecto a los servicios/productos ofrecidos. Lo anterior se puede entender como prestar servicios/producir productos con calidad. Para Evans [2], si una empresa quiere crear clientes satisfechos, primero debe identificar sus necesidades, diseñar los sistemas de producción y servicios para cumplirlas, y medir los resultados como base de la mejora.

A través de la historia se han definido diferentes teorías y modelos con respecto a la calidad, dentro de los cuales se menciona el Control Estadístico de Procesos (CEP), permitiendo a las empresas analizar sus resultados y tomar las acciones correspondientes para alcanzar los objetivos trazados.

El CEP se puede definir, según Evans [3], como “...una metodología para el seguimiento de un proceso para identificar las causas de la variación y señalar la necesidad de emprender una acción correctiva en el momento apropiado". Lo anterior permite a las organizaciones enfocar los recursos pertinentes en las acciones, con el fin de alcanzar los resultados esperados mediante el análisis y mejoramiento continuo de sus procesos.

Este artículo contextualizará al lector sobre los conceptos relacionados con el Control estadístico de procesos, y su aplicación, mediante casos de estudio, en empresas de servicios.

\section{Materiales y métodos}

Se realizó la búsqueda documental en el periodo comprendido entre los años 2004 al 2015, mediante:

-Palabras claves: Control estadístico, gráficos de control, empresas de servicios, herramientas de calidad, métodos 
estadísticos.

-Tesauros: Quality control, Service industries, Process control.

- Bases de datos:

$\begin{array}{ll}\text { - Redalyc } & \text { - Ebrary } \\ \text { - Scielo } & \text { - Proquest } \\ \text { - Ebsco } & \text { - Virtual pro }\end{array}$

\section{Resultados y análisis}

Según Kumar [4], en 1920, Walter A. Shewart, de Laboratorios Bell Telephone, fue el pionero en el campo del Control Estadístico de Procesos. Como lo define Juran [5], los procesos muestran variabilidad; así mismo, Vargas Quiñonez [6] menciona que cualquier proceso, incluida la prestación de servicios de salud y servicios educativos, produce variabilidad. De acuerdo con Fontalvo [7], la base del control estadístico es la comprensión de la variación natural de los procesos, la cual puede ser por causas comunes (azar), o a causas especiales (situaciones particulares), como se muestra en la Figura 1.

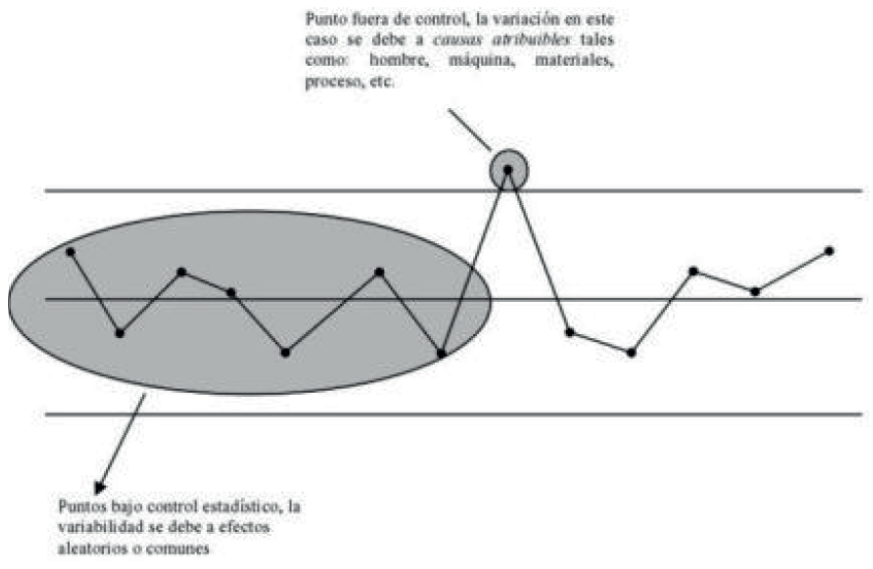

Figura 1. Control estadístico de procesos Fuente: [7]

Para González Gaya [8] las causas comunes o no asignables, y las especiales o asignables tienen las características mostradas en la Tabla I.

Tabla I. Características de las causas comunes o no asignables y las especiales o asignables

\begin{tabular}{|c|c|}
\hline Causas no asignables o comunes & Causas asignables o especiales \\
\hline Son de carácter aleatorio & $\begin{array}{c}\text { Existe un número pequeño, pero } \\
\text { cada una de ellas produce un } \\
\text { efecto fuerte. }\end{array}$ \\
\hline $\begin{array}{c}\text { Existe una gran cantidad, pero cada } \\
\text { una es de pequeña importancia }\end{array}$ & $\begin{array}{c}\text { Producen una variabilidad } \\
\text { imprevisible }\end{array}$ \\
\hline Producen variabilidad estable & $\begin{array}{c}\text { Sus efectos desaparecen al } \\
\text { eliminar la causa }\end{array}$ \\
\hline Es difícil reducir efectos & \\
\hline
\end{tabular}

Fuente: [19]
Por otro lado, Greff y Ghoshal [9] definen que las técnicas de CEP (SPC por sus siglas en inglés) incluyen:

- Cartas de control,

- Hojas de verificación,

- Diagrama de Paretto,

- Diagrama de Causa y Efecto,

- Diagrama de defectos,

- Diagrama Scatter,

- Histogramas

Zhang [10] menciona que el control estadístico de procesos (CEP) es un método para monitorear un proceso industrial a través del uso de cartas de control. Siguiendo el mismo concepto, Cuatrecasas [11] menciona que los gráficos de control se emplean en el CEP como herramienta para analizar la variabilidad de los procesos con el tiempo, ayudando a identificar las posibles causas de variación o desviación.

De acuerdo con la información anterior, Ishikawa [12] menciona que el término gráficos de control fue usado por el Dr. W.A Shewart en 1926. A su vez, Hansen [13] establece que un gráfico de control es un dibujo para determinar si el modelo de probabilidad es estable o cambia a lo largo del tiempo.

El uso de los gráficos de control está ligado con el tipo de dato que se va a analizar. Kumar [14] menciona que los cuadros de control se han clasificado por mediciones (continuos) y por atributos (discretos), dependiendo de si las observaciones sobre las características de calidad son mediciones o enumeraciones de datos. Evans [15] define los datos variables como aquellos que se miden con base en una escala continua (ejemplo: longitud, peso, tiempo y distancia). Juran [16] menciona que los datos [variables] consisten en números a lo largo de una escala de medida: 110 voltios; 182 gramos; 23,2 segundos.

\section{Tipos de gráfico}

\section{Por variables}

- Gráfico x - R. Evans [17] menciona que la gráfica x se usa para el seguimiento del centrado del proceso, mientras que el $\mathrm{R}$ se usa para el seguimiento de la variación del proceso. Kumar [18] maneja un concepto similar, indicando que el gráfico $\mathrm{X}$-bar (x) es un cuadro de control desarrollado sobre una calidad promedio, y los cuadros R (rango) están basados en el rango de valores que representan la variación en la respuesta al tiempo analizado.

- Gráfico x - s. González Gaya [19] establece que la media (x) mide la tendencia central, mientras que la desviación estándar (s) representa la dispersión de los datos. Para Evans [20], la desviación estándar de la muestra es un indicador más sensible y mejor de la variabilidad del proceso que el rango (R), especialmente para tamaños de muestras grandes. 


\section{Por atributo}

- Gráfico p. Para Ishikawa [21] este gráfico se usa cuando el número de unidades defectuosas de una muestra es como fracción de unidades defectuosas, para un tamaño de n muestras variables.

- Gráfico np. González Gaya [22] establece el gráfico para el número de unidades disconformes, cuando las muestras tomadas son todas del mismo tamaño.

- Gráfico c. Para Evans [23] este gráfico se utiliza para controlar el número total de defectos por unidad cuando el tamaño del subgrupo es constante.

- Gráfico u. Para Kumar [24], este gráfico de control se utiliza para cuando los datos de los defectos muestrales se recogen de n componentes en la muestra.

En la tabla II se muestra un resumen sobre el uso del gráfico de control, según el tipo de dato.

Tabla II. Resumen sobre el uso del gráfico de control, según el tipo de dato

\begin{tabular}{|c|c|c|}
\hline Tipo de dato & Gráfico de control & Uso \\
\hline Variable & $\mathbf{x}-\mathbf{R}$ & $\begin{array}{c}\text { Seguimiento de la media y rango del } \\
\text { proceso }\end{array}$ \\
\hline Variable & $\mathbf{x}-\mathbf{S}$ & $\begin{array}{c}\text { Seguimiento de la media y desviación } \\
\text { estándar del proceso }\end{array}$ \\
\hline Atributo & $\mathbf{P}$ & Fracción de disconformidades \\
\hline Atributo & $\mathbf{N p}$ & Número de unidades disconformidades \\
\hline Atributo & $\mathbf{C}$ & Número de disconformidades \\
\hline Atributo & $\mathbf{U}$ & Número de disconformidades / unidad \\
\hline
\end{tabular}

Ventajas y desventajas de las principales herramientas del control estadístico de procesos (CEP).

Kumar [25] presenta las siguientes ventajas y desventajas de usar un cuadro de control (gráfico de control):

\section{Ventajas}

- Muy efectiva en la prevención de defectos.

- Es una técnica probada en la mejora de la calidad y de la productividad.

- Suministra información sobre la capacidad del proceso.

- Es una buena herramienta de diagnóstico.

- Puede usarse con variables dependientes e independientes.

\section{Desventajas}

- No es una herramienta simple; todos los usuarios deben estar bien entrenados y deben participar en un programa de educación continuada.
- Los datos correctos deben recopilarse.

- Los parámetros de la herramienta (media, desviación estándar, rango) deben calcularse correctamente.

- El usuario debe tener un buen conocimiento de cómo analizar correctamente los cuadros de control.

Adicionalmente, las diferentes aplicaciones que tienen los gráficos de control se pueden considerar como una ventaja. Ishikawa [26] menciona las siguientes áreas de aplicación:

- Para control: Papel esencial del gráfico de control. El control de calidad empieza y termina con el gráfico de control.

- Para análisis: Se considera una etapa preparatoria en la cual se preparan los gráficos de control útiles para el control del proceso.

- Como gráficos: Los datos se representan en forma de gráfico de control, pero no se utilizan como tal. Aunque se representen los límites de control, estos gráficos solo se ojean.

- Para ajuste: Quiere decir, por ejemplo, cambiar la temperatura, el filo de una herramienta cortante, la composición de una materia prima, u otra condición del proceso cuando un gráfico de control indique que un proceso está fuera de control, sin buscar necesariamente la causa de la anomalía o entrar en acción para eliminarla.

- Para inspección: Utilizarlos de varias maneras desde el punto de vista de la inspección: por ejemplo, cuando un gráfico indica que hay una anomalía en un lote, y el lote se trata de diferente manera.

\section{Casos de aplicación}

El estudio permitió identificar la aplicación del control estadístico de procesos en empresas de diferentes sectores de servicios.

Servicios educativos - Escuela de Negocios Internacionales.

Según Green [27] las escuelas de negocio acreditadas por la AACSB (Association to Advance Collegiate Schools of Business International) deben implementar y mantener programas de evaluación integrales para garantizar que los servicios educativos suministrados a los estudiantes son de alta calidad. También menciona que “...los gráficos de control son desarrollados y utilizados para monitorear los procesos de prestación de servicios educativos en un esfuerzo por mantener y controlar los procesos..."

En este caso se menciona la aplicación de tres exámenes (pre test), y del examen final (pos-test) por cada grupo evaluado. En la tabla III se muestran los resultados promedio de los exámenes. 
Tabla III. Resultado promedio de los examenes

\begin{tabular}{|c|c|}
\hline Grupo 1 & \\
\hline Pre-test mean: & 39.11 \\
\hline Post-test mean: & 84.32 \\
\hline Mean difference: & +45.21 \\
\hline Significance: & Mean difference is positive and significant at .01 level \\
\hline Grupo 2 & 39.94 \\
\hline Pre-test mean: & 85.35 \\
\hline Post-test mean: & +46.41 \\
\hline Mean difference: & Mean difference is positive and significant at .01 level \\
\hline Significance: & . \\
\hline
\end{tabular}

Fuente: [29]

Green [28] menciona que el promedio objetivo para el post-test (último examen) se fija en 85. Suponiendo un tamaño de clase de 36, y que el estudiante obtiene una puntuación mínima y máxima de 70 y 100, los límites de control superior e inferior, con tres sigmas, son 82.50 y 87.50 respectivamente. El promedio del examen final (post-test) para el grupo 1 es 84.32 , y para el grupo 2 es 86,35 están dentro de los límites de control, lo cual indica que el proceso está bajo control. Las figuras 2 y 3 muestran los gráficos de control respectivos.

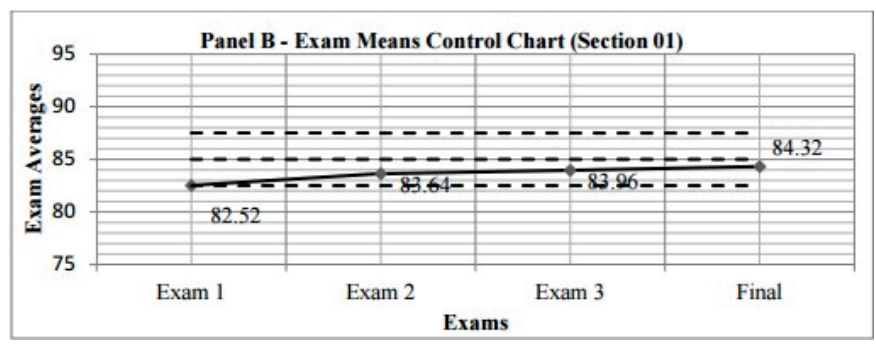

Figura 2. Gráfico de control para el resultado de los exámenes en el grupo 1 Fuente: [28]

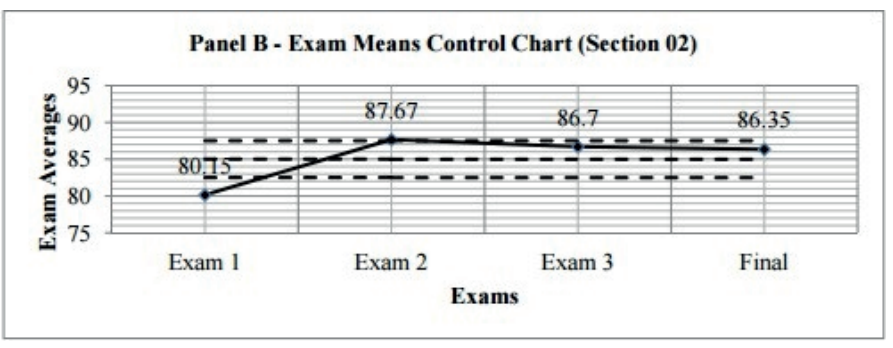

Figura 3. Gráfico de control para el resultado de los exámenes en el grupo 2 Fuente: [28]

Green [29] menciona que la segunda variable analizada fue la asistencia de los estudiantes a clase. El análisis de los datos de asistencia muestra los siguientes resultados:

Grupo 1. 86.30\%. Tasa global de asistencia. Grupo 2. 88.00\%. Tasa global de asistencia.
El promedio objetivo de asistencia a tiempo es 0,85 . Suponiendo un tamaño de la clase de 36 , los límites de control definidos son 0.67 y 1.00 . Las proporciones promedio de asistencia para los grupos 01 y 02 son 0,86 y 0,88 . Ambos están dentro de los límites de control.

Las figuras 4 y 5 muestran los gráficos de control respectivos:

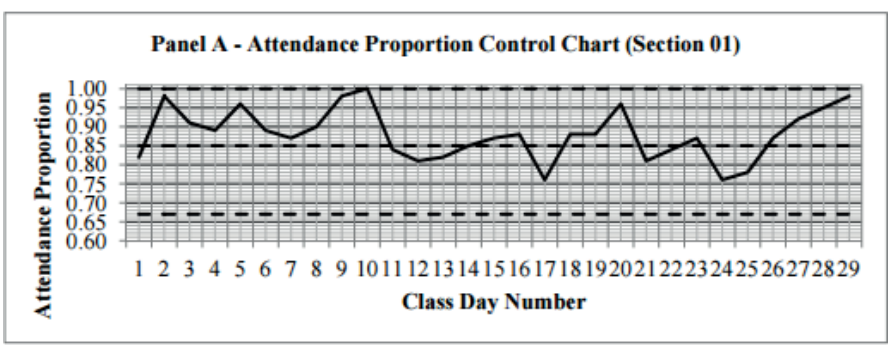

Figura 4. Gráfico de control para el resultado de la proporción de asistencia en el grupo 1

Fuente: [28]

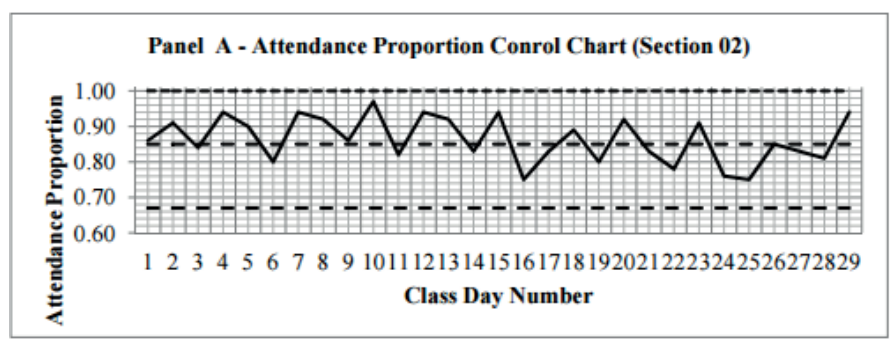

Figura 5. Gráfico de control para el resultado de la proporción de asistencia en el grupo 2

Fuente: [28]

Green [30] concluye que los gráficos de control de procesos se utilizan para supervisar los procesos que garantizan la prestación de servicios educativos de calidad, y cumplir con los requisitos establecidos, en este caso, por la AACSB.

\section{Servicios financieros - Operación en un banco comercial de Taiwan}

Tsai [31], identifica la aplicación del CEP en un banco de Taiwán. El autor menciona que hay cientos de miles de informes en la literatura que se centraron en la mejora de la calidad y la aplicación de control estadístico de procesos en la industria manufacturera, pero que algunos autores, como Wheeler, trataron el concepto del CEP no solo en producción de bienes, también en la entrega de servicios.

La investigación tiene como objetivo entender y probar si las herramientas CEP que se aplican mayoritariamente en la fabricación de los sectores productivos, son igualmente prácticas en instituciones financieras. El Banco define una hoja de ruta bastante interesante, la cual se muestra en la Figura 6. 


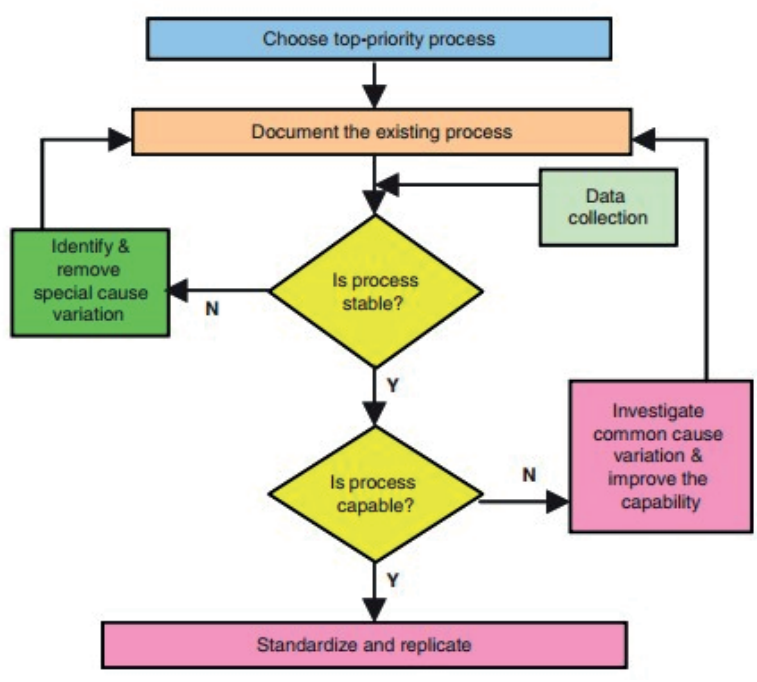

Figura 6. Hoja de ruta Fuente: [31]

Para escoger la prioridad más alta del proceso (choose top-priority process), en junio de 2004, Prudential Bank distribuyó una encuesta de satisfacción del cliente a 400 clientes corporativos, para realizar un seguimiento de la regeneración de clientes hacia los dos procesos principales de productos transfronterizos, a saber, remesas hacia el exterior $(\mathrm{O})$ y las remesas hacia el interior (IR).

En la Figura 7 se muestra el resultado de la encuesta realizada por la organización.

\begin{tabular}{|c|c|c|c|c|c|c|c|c|c|c|}
\hline & \multicolumn{2}{|c|}{ Overall } & \multicolumn{2}{|c|}{$\begin{array}{c}\text { Service } \\
\text { attitude }\end{array}$} & \multicolumn{2}{|c|}{$\begin{array}{l}\text { Trouble case } \\
\text { handling skill }\end{array}$} & \multicolumn{2}{|c|}{$\begin{array}{c}\text { Outward } \\
\text { remittance }\end{array}$} & \multicolumn{2}{|c|}{$\underset{\text { speed }}{\mathbb{R}}$} \\
\hline & 2003 & 2004 & 2003 & 2004 & 2003 & 2004 & 2003 & 2004 & 2003 & 2004 \\
\hline $\begin{array}{l}\text { Satisfied + very } \\
\text { satisfied (\%) }\end{array}$ & 81.39 & 83.98 & 82.51 & 93.35 & 84.23 & 92.58 & 79.76 & 83.39 & 70.35 & 73.25 \\
\hline $\begin{array}{r}\text { Dissatisfied + very } \\
\text { dissatisfied (\%) }\end{array}$ & 3.88 & 5.36 & 3.26 & 0.89 & 4.42 & 0.88 & 3.02 & 2.83 & 0.78 & 1.12 \\
\hline
\end{tabular}

Figura 7. Resultados encuesta de satisfacción Fuente: [31]

De ese resultado, el Banco decide enfocarse en el proceso IR como prioridad más alta. El Banco identifica las posibles causas que llevaron al resultado del proceso, las cuales se muestran en Figura 8.

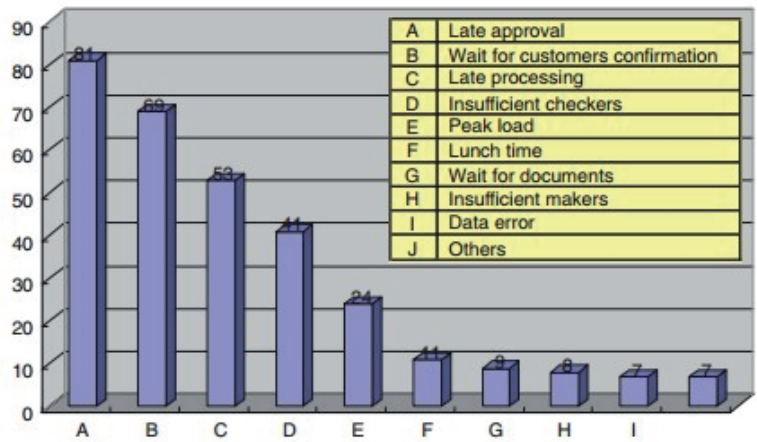

Figura 8. Las posibles causas del largo tiempo de ciclo del procesamiento de remesas interno

Fuente: [31]
Para establecer si el proceso es estable, el Banco decide tomar una muestra de 25 datos desde el 03 de septiembre de 2004 hasta el 5 de noviembre del 2004. Los datos se muestran en la Figura 9.

\begin{tabular}{|c|c|c|c|c|c|c|c|c|c|c|c|c|c|}
\hline & $9 / 30$ & $10 / 1$ & $10 / 4$ & $10 / 5$ & $10 / 6$ & $10 / 7$ & $10 / 8$ & $10 / 11$ & $10 / 12$ & $10 / 13$ & $10 / 14$ & $10 / 15$ & $10 / 18$ \\
\hline & 1 & 2 & 3 & 4 & 5 & 6 & 7 & 8 & 9 & 10 & 11 & 12 & 13 \\
\hline X1 & 202.00 & 122.00 & 455.00 & 177.00 & 36.00 & 106.00 & 400.00 & 248.00 & 20.00 & 81.00 & 110.00 & 142.00 & 89.00 \\
\hline $\mathrm{X} 2$ & 120.00 & 176.00 & 453.00 & 128.00 & 34.00 & 108.00 & 174.00 & 250.00 & 20.00 & 44.00 & 111.00 & 162.00 & 89.00 \\
\hline $\mathrm{X} 3$ & 117.00 & 43.00 & 103.00 & 128.00 & 56.00 & 11.00 & 90.00 & 43.00 & 61.00 & 51.00 & 114.00 & 62.00 & 103.00 \\
\hline $\mathrm{X} 4$ & 110.00 & 123.00 & 102.00 & 342.00 & 56.00 & 9.00 & 336.00 & 172.00 & 64.00 & 57.00 & 116.00 & 182.00 & 112.00 \\
\hline $\mathrm{X} 5$ & 117.00 & 113.00 & 135.00 & 155.00 & 138.00 & 75.00 & 279.00 & 42.00 & 25.00 & 100.00 & 116.00 & 66.00 & 114.00 \\
\hline \multirow[t]{3}{*}{$\mathrm{X} 6$} & 116.00 & 287.00 & 135.00 & 177.00 & 296.00 & 75.00 & 137.00 & 39.00 & 72.00 & 43.00 & 67.00 & 249.00 & 116.00 \\
\hline & $10 / 20$ & $10 / 21$ & $10 / 22$ & $10 / 26$ & $10 / 27$ & $10 / 28$ & $10 / 29$ & $11 / 1$ & $11 / 2$ & $11 / 3$ & $11 / 4$ & $11 / 5$ & \\
\hline & 14 & 15 & 16 & 17 & 18 & 19 & 20 & 21 & 22 & 23 & 24 & 25 & \\
\hline $\mathrm{X} 1$ & 49.00 & 226.00 & 327.00 & 130.00 & 90.00 & 100.00 & 72.00 & 172.00 & 51.00 & 43.00 & 111.00 & 8.00 & \\
\hline $\mathrm{X} 2$ & 137.00 & 111.00 & 203.00 & 21.00 & 56.00 & 101.00 & 87.00 & 163.00 & 67.00 & 9.00 & 67.00 & 13.00 & \\
\hline $\mathrm{X} 3$ & 249.00 & 41.00 & 126.00 & 144.0 & 83.00 & 97.00 & 239.00 & 32.00 & 111.00 & 60.00 & 79.00 & 54.00 & \\
\hline $\mathrm{X} 4$ & 550.00 & 154.00 & 118.00 & 144.00 & 9.00 & 142.00 & 192.00 & 82.00 & 273.00 & 56.00 & 24.00 & 48.00 & \\
\hline X5 & 168.00 & 153.00 & 22.00 & 155.00 & 364.00 & 98.00 & 19.00 & 425.00 & 56.00 & 130.00 & 15.00 & 141.00 & \\
\hline X6 & 11.00 & 276.00 & 116.00 & 153.00 & 241.00 & 8.00 & 19.00 & 35.00 & 128.00 & 28.00 & 100.00 & 100.00 & \\
\hline
\end{tabular}

Figura 9. Datos generados desde el $30 / 9$ hasta $5 / 11$ Fuente: [31]

Con los datos que se obtuvieron, y mediante el uso del gráfico de control X - R, se determina que el proceso no es estable. El resultado se muestra en la Figura 10.
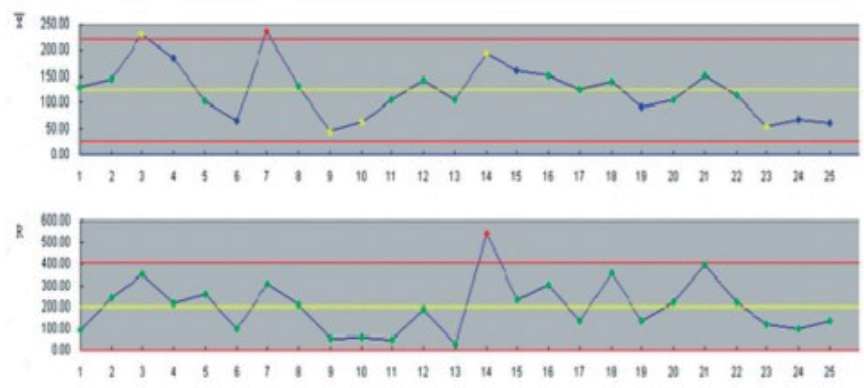

Figura 10. Gráfico de control XR datos generados desde el 30/9 hasta 5/11 Fuente: [31]

Dado que el proceso no es estable, se identifican las causas especiales que impactaron el proceso, y se determinan las soluciones para eliminarlas. Después de implementar las soluciones identificadas, el Banco vigila el comportamiento del proceso entre el 5 de noviembre y el 10 de diciembre, lo cual se muestra en la Figura 11.

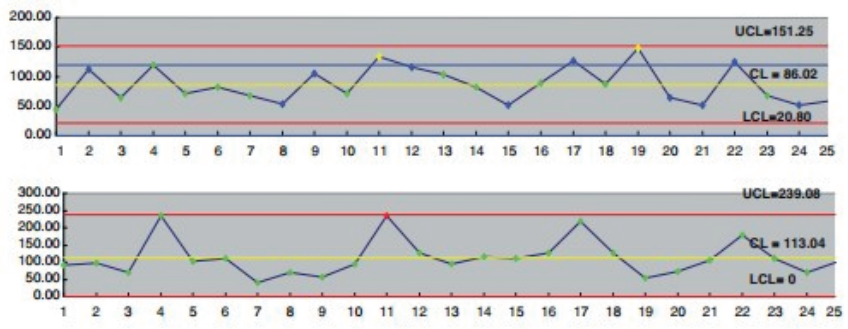

Figura 10. Gráfico de control XR - datos tomados del 5/11 al 10/12 Fuente: [31] 


\section{Como conclusión del caso de estudio, Tsai [30] define:}

La hoja de ruta de SPC fue desarrollada y probada usando datos empíricos de varias unidades de funcionamiento de un banco comercial de Taiwán. Los dos casos se ensayaron, y los resultados muestran que el modelo teórico es eficaz y viable.

Debido a la aplicación de SPC con resultados positivos del caso elegido de una operación de remesas transfronteriza, el banco se esforzó continuamente por los programas de calidad en los años siguientes en campos tales como el procesamiento de préstamos hipotecarios, vehículo préstamo de descenso, transacciones manejadas por el mostrador de servicio de sucursales del banco, etc.

\section{Conclusiones}

- El control estadístico de procesos (gráficos de control) permite identificar la(s) causa(s) de variación del proceso, aplicando el gráfico de control respectivo, y tomar la(s) acción(es) correspondiente(s).

- Existe un gráfico de control para analizar el comportamiento del proceso, independientemente el tipo de variable (continua o discreta) elegida por la organización para ser analizada.

- El control estadístico de procesos es un concepto que se aplica desde hace un siglo, aproximadamente, sin embargo, su uso ha estado relacionado con empresas del sector manufactura. Este trabajo investigativo permitió identificar empresas de diferentes sectores de servicios que aplican el control estadístico de procesos (gráficos de control) para conocer la variación de $\mathrm{su}(\mathrm{s}) \operatorname{proceso}(\mathrm{s}), \mathrm{la}(\mathrm{s})$ causa(s) de esta variación, y establecen el(los) plan(es) de acción correspondiente(s) para estabilizarlo(s) y mejorar su capacidad.

- Los aspectos clave en la implementación del control estadístico de procesos (gráficos de control) son: La competencia del personal que genera el gráfico y/o analiza el resultado; y la confiabilidad de los datos que analizarán.

\section{Referencias}

[1] Zeithaml, Valarie A., Parasuraman, A., and Berry, Leonard L. El punto de vista de del consumidor sobre la calidad de los servicios. En: Calidad total en la gestión de servicios. [En línea]. España: Ediciones Díaz de Santos, 2007. p. 17. ISBN: 9788479780616. [Consultado 04, abril, 2016]. Disponible en: http://site.ebrary.com/lib/biblioamericasp/reader.action?docI$\mathrm{D}=10184529 \&$ ppg $=32$

[2] Evans, James R., Lindsay, William R. Introducción a la calidad. En: Administración y Control de la .Calidad. Editor: Sergio R. Cervantes González. 7 ed. México D.F: Cengage Learning Editores, 2008. p 154. ISBN-13: 978-970-686-836-7

[3] Evans, James R., Lindsay, William R. Control Estadistico de Procesos. En: Administración y Control de la Calidad. Editor: Sergio R. Cervantes González. 7 ed. México D.F: Cengage Learning Editores, 2008. p 712. ISBN-13: 978-970-686-836-7.

[4] Kumar, Dhirendra. Controlar. En: Six Sigmas Las mejores prácticas. Traductor: Jaime Valencia Villa. Bogotá: 3R Editores, 2009. p. 389. ISBN: 978-958-30-3090-1

[5] Juran, Joseph M. Desarrollo del proceso. En: Juran y la planificación para la calidad. [En línea]. España: Ediciones Díaz de Santos, 2008. p. 164. ISBN: 9788487189371. [Consultado 04, marzo, 2016]. Disponible en: http://site.ebrary.com/lib/biblioamericasp/reader.action?docID $=10204125 \& p p g=164$

[6] Vargas Quiñones, Martha Elena, and Aldana de Vega, Luz Ángela. La ruta del cliente y herramientas utilizadas en la gestión de la calidad y el servicio. En: Calidad y servicio: conceptos y herramientas. [en línea]. 2. ed. Ecoe Ediciones, 2011. p. 128. ISBN: 9781449276041. [Consultado 04, marzo, 2016] Disponible en: http://site.ebrary.com/lib/biblioamericas$\mathrm{p} /$ detail.action?docID $=10552805$.

[7] Fontalvo Herrera, Tomás José. Sistema de gestión de la calidad. En: La gestión avanzada de la calidad: metodologías eficaces para el diseño, implementación y mejoramiento de un sistema de gestión de la calidad. [En línea]. Colombia: Corporación para la gestión del conocimiento ASD 2000, 2006. p. 44. ISB: 9789583389535. [Consultado 04, marzo, 2016]. Disponible en: http://site.ebrary.com/lib/biblioamericasp/reader.action?docID $=10179523 \& p p g=6$

[8] González Gaya, Cristina, Domingo Navas, Rosario, and Pérez, Miguel Ángel Sebastián. La mejora de la calidad. En: Técnicas de mejora de la calidad: [En línea]. UNED - Universidad Nacional de Educación a Distancia, 2000. p. 26. ISBN: 9788436266412. [Consultado 04, marzo, 2016]. Disponible en: http://site.ebrary.com/lib/biblioamericasp/reader.action?docI$\mathrm{D}=10803889 \&$ ppg $=72 \#$.

[9] Greeff, Gerhard Ghoshal, Ranjan. Practical E-Manufacturing and Supply Chain Management. [En línea]. p 205. Knovel. Disponible en http://app.knovel.com/hotlink/toc/id:kpPEMSC$\mathrm{M} 0 \mathrm{~B} /$ practical-e-manufacturing/practical-e-manufacturing. Texto original

[10] Zhang, Peng. Advanced Industrial Control Technology. Knovel. [En línea]. 2010. Disponible en: http://app.knovel.$\mathrm{com} / \mathrm{hot} \mathrm{ink} / \mathrm{t}$ oc/id: kp A IC T 0003 / advanced-in dustrial-control/advanced-industrial-control.

[11] Cuatrecasas, Lluís. La Gestión de la Calidad y su mejora. Herramientas. En: Gestión integral de la calidad: implantación, 
control y certificación. [En línea]. 3 ed. España: Ediciones Gestión 2000, 2009. p. 77. ISBN: 9781413579703 . [Consultado 04, marzo, 2016]. Disponible en: http://site.ebrary.com/lib/biblioamericasp/reader.action?docID $=10317466 \& p p g=10$

[11] Ishikawa, Kaoru. El enfoque estadístico y algunas herramientas estadísticas sencillas. En: Introducción al control de calidad. [En línea]. España: Ediciones Díaz de Santos, 2007. p. 120. ISBN: 9788479781729. [Consultado 04, marzo, 2016]. Disponible en: http://site.ebrary.com/lib/biblioamericasp/reader.action?docID=10198498\&ppg=134\#

[13] Hansen, Bertrand L., and Ghare, Prabhakar M. Control estadistico de procesos. En: Control de calidad: teoría y aplicaciones. [En línea]. España: Ediciones Díaz de Santos, 2008. p. 91. ISBN: 9788487189319. [Consultado 04, marzo, 2016]. Disponible en: http://site.ebrary.com/lib/biblioamericasp/reader.action?docID $=10249536 \& p p g=108$

[14] Kumar, Dhirendra. Controlar. En: Six Sigmas Las mejores prácticas. Traductor: Jaime Valencia Villa. Bogotá: 3R Editores, 2009. p. 392. ISBN: 978-958-30-3090-1

[15] Evans, James R., Lindsay, William R. Control estadìstco de procesos. En: Administración y Control de la Calidad. Editor: Sergio R. Cervantes González. 7 ed. México D.F: Cengage Learning Editores, 2008. p 718. ISBN-13: 978-970-686-836-7

[16] Juran, Joseph M. Desarrollo del proceso. En: Juran y la planificación para la calidad. [En línea]. España: Ediciones Díaz de Santos, 2008. p. 162. ISBN: 9788487189371. [Consultado 04, marzo, 2016]. Disponible en: http://site.ebrary.com/lib/biblioamericasp/reader.action?docID=10204125\&ppg=164

[17] Evans, James R., Lindsay, William R. Control estadìstco de procesos. En: Administración y Control de la Calidad. Editor: Sergio R. Cervantes González. 7 ed. México D.F: Cengage Learning Editores, 2008. p 718. ISBN-13: 978-970-686-836-7.

[18] Kumar, Dhirendra. Controlar. En: Six Sigmas Las mejores prácticas. Traductor: Jaime Valencia Villa. Bogotá: 3R Editores, 2009. p. 393. ISBN: 978-958-30-3090-1

[19] González Gaya, Cristina, Domingo Navas, Rosario, and Pérez, Miguel Ángel Sebastián. Técnicas básicas de mejora de la calidad. 7H. En: Técnicas de mejora de la calidad: [En línea]. UNED - Universidad Nacional de Educación a Distancia, 2000. p. 73. ISBN: 9788436266412. [Consultado 04, abril, 2016]. Disponible en: http://site.ebrary.com/lib/biblioamericasp/reader.action?docID $=10803889 \& p p g=72 \#$.

[20] Evans, James R., Lindsay, William R. Control estadístico de procesos. En: Administración y Control de la Calidad. Editor: Sergio R. Cervantes González. 7 ed. México D.F: Cengage Learning Editores, 2008. p 737. ISBN-13: 978-970-686-836-7
[21] Ishikawa, Kaoru. Preparación y uso de los gráficos de control. En: Introducción al control de calidad. [En línea]. España: Ediciones Díaz de Santos, 2007. p. 165. ISBN: 9788479781729. [Consultado 04, abril, 2016]. Disponible en: http://site.ebrary.com/lib/biblioamericasp/reader.action?docI$\mathrm{D}=10198498 \&$ ppg $=134 \#$

[22] González Gaya, Cristina, Domingo Navas, Rosario, and Pérez, Miguel Ángel Sebastián. Técnicas básicas de mejora de la calidad. 7H. En: Técnicas de mejora de la calidad: [En línea]. UNED - Universidad Nacional de Educación a Distancia, 2000. p. 75. ISBN: 9788436266412 [Consultado 04, abril, 2016]. Disponible en: http://site.ebrary.com/lib/biblioamericasp/reader.action?docID=10803889\&ppg=72\#.

[23] Evans, James R., Lindsay, William R. Control estadístico de procesos. En: Administración y Control de la Calidad. Editor: Sergio R. Cervantes González. 7 ed. México D.F: Cengage Learning Editores, 2008. p 753. ISBN-13: 978-970-686-836-7

[24] Kumar, Dhirendra. Controlar. En: Six Sigmas Las mejores prácticas. Traductor: Jaime Valencia Villa. Bogotá: 3R Editores, 2009. p. 415. ISBN: 978-958-30-3090-1

[25] Kumar, Dhirendra. Controlar. En: Six Sigmas Las mejores prácticas. Traductor: Jaime Valencia Villa. Bogotá: 3R Editores, 2009. p. 391. ISBN: 978-958-30-3090-1

[26] Ishikawa, Kaoru. El enfoque estadístico y algunas herramientas estadísticas sencillas. En: Introducción al control de calidad. [En línea]. España: Ediciones Díaz de Santos, 2007. p. 127. ISBN: 9788479781729. [Consultado 04, abril, 2016]. Disponible en: http://site.ebrary.com/lib/biblioamericasp/reader.action?docID=10198498\&ppg=134\#

[27] Green Jr, Kenneth W. Toms, Lisa. Stinson, Terrye. En: Academy of Educational Leadership Journal. [en línea]. 2012. Número 2. Volumen 16. P. 33. [Consultado 15, abril, 2016]. Disponible http://web.a.ebscohost.com.ez.uamerica.edu.co/e-

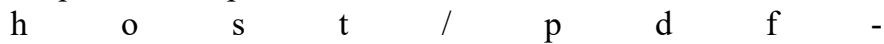
viewer/pdfviewer?sid=694907b2-2a98-442e-a71d-48d $292 \mathrm{a} 73 \mathrm{dc} 4 \% 40$ sessionmgr $4003 \& \mathrm{vid}=1 \&$ hid $=4104$

[28] Green Jr, Kenneth W. Toms, Lisa. Stinson, Terrye. En: Academy of Educational Leadership Journal. [en línea]. 2012. Número 2. Volumen 16. P. 42. [Consultado 15, abril, 2016]. Disponible http://web.a.ebscohost.com.ez.uamerica.edu.co/e$\mathrm{h} \quad \mathrm{o} \quad \mathrm{s} \quad \mathrm{t} \quad / \quad \mathrm{p} \quad \mathrm{d} \quad \mathrm{f}$ viewer/pdfviewer?sid=694907b2-2a98-442e-a71d-48d $292 \mathrm{a} 73 \mathrm{dc} 4 \% 40$ sessionmgr4003\&vid=1\&hid $=4104$

[29] Green Jr, Kenneth W. Toms, Lisa. Stinson, Terrye. En: Academy of Educational Leadership Journal. [en línea]. 2012. Número 2. Volumen 16. P. 40. [Consultado 15, abril, 2016]. Disponible http://web.a.ebscohost.com.ez.uamerica.edu.co/ehost/pdfviewer/pdfviewer?sid=694907b2-2a98-442e-a71d-48 
d292a73dc4\%40sessionmgr4003\&vid=1\&hid=4104

[30] Green Jr, Kenneth W. Toms, Lisa. Stinson, Terrye. En: Academy of Educational Leadership Journal. [en línea]. 2012. Número 2. Volumen 16. p. 43. [Consultado 15, abril, 2016]. Disponible http://web.a.ebscohost.com.ez.uamerica.edu.co/ehost/pdfviewer/pdfviewer?sid=694907b2-2a98-442e-a71d-48 d292a73dc4\%40sessionmgr4003\&vid=1\&hid=4104

[31] Tsai Ming-Ching, Chao Ou-Yang. Improving a commercial bank's operation performance through statistical process control. En: Journal of the Chinese Institute of Industrial Engineers. [en línea]. 2010. Vol. 27 Issue 3, p. 226-236. [Consultado 15, abril, 2016]. Disponible: http://web.b.ebscohost.com/ehost/pdfviewer/pdfviewer?sid=b340d260-7f17-44ec-95 cc-811005afcad3\%40sessionmgr106\&vid=1\&hid $=128$

[32] TSAI Ming-Ching, Chao Ou-Yang. Improving a commercial bank's operation performance through statistical process control. En: Journal of the Chinese Institute of Industrial Engineers. [en línea]. 2010. Vol. 27 Issue 3, p. 233. [Consultado 15, abril, 2016]. Disponible: http://web.b.ebscohost.com/ehost/pdfviewer/pdfviewer?sid=b340d260-7f17-44ec-95cc- 